\title{
SPATIAL AND TEMPORAL CHANGES OF HABITAT QUALITY IN JIANGSU YANCHENG WETLAND NATIONAL NATURE RESERVE - RARE BIRDS OF CHINA
}

\author{
ZHANG, H.-B. ${ }^{1 *}-$ WU, F.-E. ${ }^{1}-$ ZHANG, Y.-N. ${ }^{2}-$ HAN, S. ${ }^{1}-$ LIU, Y.-Q. ${ }^{1}$ \\ ${ }^{I}$ School of Urban and Planning, Yancheng Teacher's University, Yancheng 224007, China \\ (e-mail:yctuzhanghb@163.com-H.B.Zhang,243178454@qq.com-F.E.Wu, \\ hanshuang412@163.com-S.Han, liuyuqing02102123@163.com-Y.Q.Liu) \\ ${ }^{2}$ Administrative Office of Jiangsu Yancheng National Nature Reserve Rare Birds \\ Yancheng 224057, China \\ (e-mail:535511794@qq.com) \\ ${ }^{*}$ Corresponding author \\ e-mail: yctuzhanghb@163.com; phone:+86-133-7526-7876 \\ (Received 26 $6^{\text {th }}$ Jan 2019; accepted $8^{\text {th }}$ Mar 2019)
}

\begin{abstract}
Taking the core area of Jiangsu Yancheng Wetland National Nature Reserve as a case, spatial and temporal changes of habitat quality were analyzed from the viewpoint of Red-crowned crane (Grus japonensis (Statius Müller, 1776)), and other rare birds. The results showed that the area of mudflats and Suaeda salsa marshes decreased, the area of Spartina alterniflora marshes, Phragmites australis marshes, aquaculture ponds and roads increased during 1983 to 2011. The value of habitat degradation index increased from 0.006 to 0.024 during 1983 to 2011, which meant the habitat degradation was becoming more serious. The spatial distribution of habitat degradation had been a distinct longitudinal band from an obvious lateral belt. The value of habitat quality index decreased from 0.863 in 1983 to 0.742 in 2011, which meant the habitat quality became worse due to the expansion of aquaculture ponds and Spartina alterniflora. Based on 1983, the value of habitat scarcity index increased from -0.10 to 0.173 during 1997 to 2011, indicating that the ecosystem function was declining. The values of habitat scarcity index of Suaeda salsa marsh and mudflat were obviously rising and were also the highest which were 0.628 and 0.637 in 2011, respectively. It indicated that Suaeda salsa marsh and mudflat became more and more scarce. They needed more protection and restoration.
\end{abstract}

Keywords: landscape change, habitat quality, spatial differentiation, InVEST, coastal wetland

\section{Introduction}

Habitat quality is one of the important service functions of the ecosystem and the objective existence of the essential attributes of the ecological environment (Xiao, 2011). However, with the increase of human disturbance, especially the change of land use mode, intensity and pattern caused by large-scale human activities, it had a heavy impact on the quality of biological habitat, and even affected the biodiversity of the entire region, and even subsequently the planet (Zhong et al., 2017). Therefore, the study of regional habitat quality was of great significance for maintaining regional ecosystem stability and improving ecosystem functions.

On the whole, the habitat quality assessment research can be divided into two categories. First, the habitat quality assessment based on on-the-spot investigation was mostly limited to a small scale, such as small cities, rivers, nature reserves and so on. Belt transect method or quadrat method was often used to investigate animals and plants, so as to obtain parameters related to habitat quality, constructing an assessment system and using certain mathematical methods to evaluate (Wu et al., 2015), which was mainly used 
to predict the spatial distribution of habitat or select location of nature reserves in the future, which can analyze the spatial distribution of species and habitat conditions, and assess the suitability of the species habitat (Chen et al., 2016). For example, Guo et al. (2016) evaluated the regional habitat quality of Zagunao river valley in the upstream of Minjiang River by using Analytic Hierarchy Process (AHP) according to 9 factors such as topography, soil, water and vegetation. However, this method is time-consuming and labor-consuming, making it difficult to observe for a long time.

The second is the index system model based on landscape pattern. With the widespread use of remote sensing and Geographic Information System (GIS) technology, integrating the landscape pattern and the distribution of threat sources into the habitat quality model, establishing a structural spatial display model has become a new research focus, is suitable for large and medium-sized research on the spatial and temporal changes of habitat quality, and is the basis for the identification, definition and conservation of ecosystem health (Gong et al., 2018). Artificial Intelligence for Ecosystem Services (ARIES), Multiscale Integrated Models of Ecosystem Services (MIMES) and Integrated Valuation of Ecosystem Services and Tradeoffs (InVEST) models have done creative research in this respect, among which the InVEST model is the most mature and most widely used ecological function evaluation model at present, and the method of evaluating habitat quality based on habitat threaten is widely used (Bhagabati et al., 2014). Terrado et al. (2016) did a comparative analysis of the calculation results of habitat quality module in model with the results of biodiversity observation. Research showed that there was a significant correlation between them, proving the reliability of the InVEST model. Chu et al. (2015) studied the changes of landscape pattern and habitat quality in Liaoning coastal zone from 2000 to 2010 by using the InVEST model, pointing out that reclamation of wetlands and urbanization expansion were the main reasons for the degradation of habitat quality. Wang (2016) evaluated the changes of habitat quality in Sanjiangyuan from 2000 to 2010 based on the InVEST model, and pointed out that the improvement of habitat quality in Sanjiangyuan in the past 10 years was a manifestation of the effectiveness of ecological restoration and construction. Bai et al. (2015) studied the changes of habitat quality in Minjiang River Basin from 2000 to 2010 by using the InVEST model, and pointed out that the interference of roads, industrial land, residential areas and mining sites were the main driving factors for the changes of habitat quality.

Yancheng Coastal Wetland is located in the central coast of Jiangsu Province of China, and is one of the most typical and representative distribution areas of muddy coastal wetlands in China and even the world, integrating intertidal beaches, tides, rivers, salt marshes, Phragmites australis marshes and Spartina alterniflora marshes. Over the years, under the dual influence of nature and human activities, especially under the highfrequency and high-intensity human production activities, the natural wetland area has been reduced and biodiversity has been lost, and the problem of ecological environment quality has become increasingly prominent, which has become one of the current focus issues, especially the study of suitability of Red-crowned Crane habitat. Sun et al. (2011) and $\mathrm{Ou}$ et al. (2015) pointed out the impact of changes in wetland landscape structure and connectivity under the influence of human activities on the habitat quality, especially on the habitat suitability of Red-crowned Cranes. Liu et al. (2016) studied the population dynamics of Red-crowned Cranes and their selection of habitat in Yancheng coastal wetland. Wang et al. (2015) selected nine ecological factors such as land use status, plant coverage, soil environment, biomass, water quality, water supply, biodiversity, wetland threated status and annual growth rate of wetland degradation, taking GIS technology, 
and the ecological suitability of nature reserves was analyzed quantitatively by using AHP and factor weighted superposition. However, the study on the spatial differentiation of habitat quality with long time series, quantification and visualization is rarely reported in Yancheng coastal wetland. Therefore, taking the core area of Jiangsu Yancheng Wetland National Nature Reserve Rare Birds as an example, this paper studied the spatial and temporal changes of the habitat quality of the core area from 1983 to 2011 by using the habitat quality module in the InVEST model, and analyzed the impact of landscape changes on the habitat quality, which can provide a reference for the construction and management of Yancheng Coastal Wetland.

\section{Material and methods}

\section{Study area}

Yancheng coastal wetland is located in the central coastal area of Jiangsu Province of China, facing the Yellow Sea in the east. The coastline is $582 \mathrm{~km}$ long and the wetland area is $4.53 \times 10^{5} \mathrm{hm}^{2}$. It is the largest tidal flat coastal wetland in the Asian continent. The nature reserve is located in the transition zone between subtropical and warm temperate zone. The monsoon is remarkable and the climate is mild. The annual precipitation is about $1000 \mathrm{~mm}$. Jiangsu Yancheng Wetland National Nature Reserve - Rare Birds $\left(32^{\circ} 20^{\prime} \mathrm{N} \sim 34^{\circ} 37^{\prime} \mathrm{N}, 119^{\circ} 29^{\prime} \mathrm{E} \sim 121^{\circ} 16^{\prime} \mathrm{E}\right)$ was established in 1983. It was upgraded to a national nature reserve in 1992. It is an important member of World Biosphere Reserve, Northeast Asia Crane Protection Network and East Asia--Australian Wader Migration Network. It has been entered into the International List of Important Wetlands. The study selected the core area of Jiangsu Yancheng Wetland National Nature Reserve $\bullet$ Rare Birds (Fig. 1) as the case area. The core area is to Xinyanggang River in the north, to Doulonggang River in the south, to the seawall road in the west, and to edge of mudflats ( $-3 \mathrm{~m}$ isobath) in the east. It is a typical silt-type tidal flat wetland with a total area of $1.92 \times 10^{4} \mathrm{hm}^{2}$. The landscape types in the core area are divided into natural wetlands, constructed wetlands and non-wetlands. Natural wetlands include Phragmites australis marsh, Spartina alterniflora marsh, Suaeda salsa marsh, mudflat and river. Constructed wetlands are culture ponds. Non-wetlands are roads.

\section{Data sources}

We used MSS image in 1983 (M4119037_037119831114), TM image in 1997 (P119737_5t19920607) and ETM image in 2011 (L71119037_03720110924) as the data sources. At the same time, we also used 1:200,000 geomorphic map, 1:200,000 vegetation map and 1:200,000 land use map of Xinyangang River estuary in "atlas of comprehensive survey of coastal and tidal marsh resources in China - fascicule of Jiangsu" which was published by the State Oceanic Administration of China and the State Bureau of Surveying and Mapping of China in 1988.

In ENVI 4.7, we first removed the trips 0n the image in 2011 by using the stripper tool. Then, we used the FLAASH module to perform atmospheric correction on remote sensing images. After atmospheric correction, we performed geometric correction of remote sensing images through GPS positioning in the field, in which the correction accuracy was less than 0.5 pixels. We classified remote sensing images in 1983 and 1997 by using the maximum likelihood method in supervised classification. Based on the principal component analysis, we combined the multispectral band with the panchromatic band for 
the image in 2011 (Serious phenomenon of foreign body with spectrum). We used a combination of unsupervised classification and decision tree classification, and used survey data of field to verify, so that computer interpretation and visual interpretation could be combined to achieve image interpretation accuracy of more than $95 \%$. We produced a series of landscape types of the study area in 1983, 1997, and 2011 by ArcGIS.

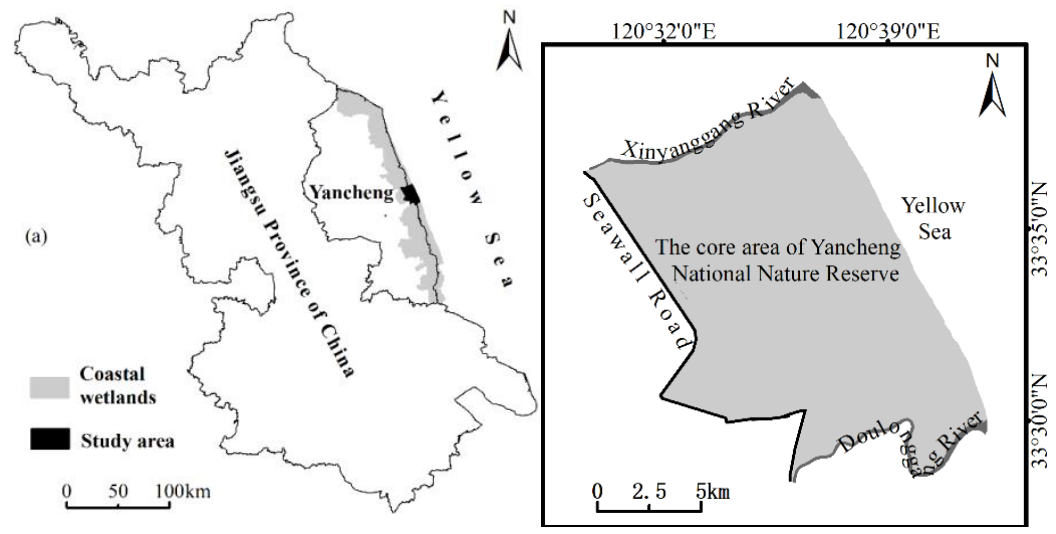

Figure 1. Location and scope of the study area. (a) The study area is located at the core zone of Yancheng National Nature Reserve, which is situated on the center of the coastal of Jiangsu Province, China. (b) The scope of the study area

\section{Methods}

The InVEST model was developed by Stanford university, the Nature Conservancy (TNC), the World Wildlife Fund (WWF) and other research institutions to evaluate Ecosystem service functions and their economic value, and support Ecosystem management and decision-making (Hall et al., 1997; Sharp et al., 2017; Zhang et al., 2017).

Habitat Quality Module in InVEST 3.2 was a combination of the landscape type map and the threat factors of biodiversity to generate a map of habitat quality. The Habitat Quality Module was based on the type of landscape that had different levels of sensitivity to different threat factors. It clearly characterized the response of various landscape types to threat factors and assessed the interaction between threat factors. The better the habitat quality was, the higher the degree of biodiversity conservation would be in the region. The more frequent human activities were, more threat factors there were, and more changes the landscape had, the greater impact on habitat quality it would have. Therefore, using the Habitat Quality Module could effectively analyze the relationship between landscape changes and habitat quality. The basic data required for the operation of the Habitat Quality Module included the map of landscape type, the map of threat factors, the influence distance table of threat factors and the table of sensitivity of landscape types to threat factors (Nelson et al., 2009; Goldstein et al., 2012; Mdk et al., 2013; Chaplinkramer et al., 2015; Zhang et al., 2016; Zhao et al., 2017).

\section{Map of landscape types}

Taking the series of landscape maps of the study areas in 1983, 1997 and 2011 as the basic data, and choosing the map of 1983 as the baseline. We also set the unified grid size to $30 \mathrm{~m} \times 30 \mathrm{~m}$. 


\section{Threat factors}

Based on the landscape type of Yancheng coastal wetlands, we selected aquaculture ponds, rivers, roads and Spartina alterniflora (Invasive species) that were affected by human factors greatly as threat factors. Since there were no aquaculture ponds in the study area in 1983, the pond was not used as a threat factor in 1983. We referred to the relevant literature (Yuan et al., 2009; Sun et al., 2011; Meng et al., 2012; Yang et al., 2014), combined with the characteristics of the study area, set the maximum influence distance of the threat factors, the weight of the threat factors, and the linear correlation of the decline as shown in Table 1.

Table 1. Attribute table of ecological threat factors

\begin{tabular}{c|c|c|c}
\hline Threat factors & Maximum impact distance $\mathbf{( k m )}$ & Weight & Linear correlation of regression \\
\hline Aquaculture pond & 1 & 0.6 & 0 \\
River & 3 & 0.4 & 1 \\
Road & 5 & 0.6 & 1 \\
Spartina alterniflora & 3 & 0.8 & 0 \\
\hline
\end{tabular}

A table of threat factors in the model, includes the relative impact of threat factors on habitat types and the distance at which habitat degradation occurs. There are two kinds of calculation formulas for the influence distance of threat factor, such as Eqs. 1 and 2.

$$
\begin{gathered}
i_{r x y}=1-\frac{d_{x y}}{d_{r x y}} \text { if linear } \\
i_{r x y}=\exp \left(-\frac{2.99}{d_{r x y}}\right) \text { if exponential }
\end{gathered}
$$

In Equations 1 and 2, $i_{r x y}$ represents the degree of influence of the threat factors $\boldsymbol{r}$ in the grid unit $\boldsymbol{y}$ on the habitat grid unit $\boldsymbol{x} ; d_{x y}$ represents the distance between the two grid units $\boldsymbol{x}, \boldsymbol{y}$; and $d_{r x y}$ represents the maximum influence distance of the threat factors (Sharp et al., 2017).

\section{Sensitivity of landscape types to threat factors}

Habitat suitability was bigger if it was closer to the original ecological system. Based on the function of maintaining the stability of the coastal wetland ecological environment, as well as the foraging and habitat environment standards for rare species of Red-crowned crane, we set the highest habit suitability was 1 , the lowest was 0 .

Different landscape types had different sensitivities to threat factors. Compared with the artificial landscape, the natural landscape had significantly weaker anti-interference ability to the threat factors and had greater sensitivity. We set the sensitivity range between 0 and 1 , and the higher the sensitivity was, the larger the value would be. If the landscape type was not sensitive to the threat factors, it was represented by 0 , and if it was extremely sensitive to the threat factors, it was represented by 1 (Bao et al., 2015; Li et al., 2016; 
Zhang et al., 2018). The sensitivity index of each landscape type to the threat factors in the study area was shown in Table 2.

Table 2. Habitat suitability and sensitivity to threat factors of different landscape types

\begin{tabular}{c|c|c|c|c|c}
\hline Landscape types & Habitat suitability & Culture pond & River & Road & Spartina alterniflora \\
\hline Aquaculture pond & 0.5 & 0 & 0.5 & 0 & 0.5 \\
River & 0.5 & 0.5 & 0 & 0.8 & 0.5 \\
Mudflat & 0.8 & 0.5 & 0.3 & 0.3 & 0.8 \\
Road & 0 & 0 & 0 & 0 & 0 \\
Phragmites australis marsh & 1 & 0.3 & 0.6 & 0.6 & 0.5 \\
Spartina alterniflora marsh & 0.5 & 0.5 & 0.5 & 0.7 & 0 \\
Suaeda salsa marsh & 1 & 0.5 & 0.8 & 0.5 & 0.8 \\
\hline
\end{tabular}

\section{Habitat degradation index}

The degree of habitat degradation was closely related to the sensitivity of landscape type to threat factors, the number of threat factors, the influence distance of threat factors and the weight of threat factors. The model assumed that within a certain range, the closer the habitat to the threat factor was, the higher the sensitivity and the habitat degradation index would be. The formula for calculating the habitat degradation was as follows.

$$
D_{x j}=\sum_{r=1}^{R} \sum_{y=1}^{y_{r}}\left(\frac{w_{r}}{\sum_{r=1}^{R} w_{r}}\right) r_{y} i_{r x y} \beta_{x} s_{j r}
$$

In Equation 3, $D_{x y}$ is the habitat degradation index, which characterizes the degree of habitat degradation; $\mathrm{R}$ refers to the number of threat factors; $y_{r}$ refers to the number of grid cells on the threat factor layer; $w_{r}$ refers to the weight value of the threat factor; $r_{y}$ refers to the number of raster unit threat factors in the layer; $\beta_{x}$ refers to the degree of protection; $s_{j r}$ refers to the sensitivity of the threat factor, and the value range is from 0 to 1 .

\section{Habitat quality index}

Habitat quality Index depends on habitat degradation index and habitat suitability. In general, habitat quality index increases with the increase of habitat suitability and decreases with the increase of habitat degradation Index. Therefore, the more frequent the landscape type changes were and larger the scope was, the higher the degree of habitat damage would be, greater the adverse impact on species survival and reproduction it would have, and worse the habitat quality would be. The higher the habitat suitability was and the better the habitat environment of the species was, the higher the habitat quality would be. The habitat quality index was calculated as follows.

$$
Q_{x j}=H_{j}\left(1-\frac{D_{x j}^{Z}}{D_{x j}^{Z}+k^{Z}}\right)
$$


In Equation 4, $Q_{x j}$ refers to the habitat quality index; $H_{j}$ represents the habitat suitability; $D_{x j}$ is the degree of habitat degradation; $k$ is the half-saturation coefficient, we set it to 15 in the study (It is half the raster resolution). $z$ is generally set to 2.5 .

\section{Habitat scarcity index}

Habitat scarcity could reflect the scarcity of the overall habitat of the region and the scarcity of a certain landscape type. The more the habitat scarce was, the more protected it should be. The higher the habitat scarcity index was, the lower the scarcity degree would be. It could be calculated as follows.

$$
\begin{gathered}
R_{j}=1-\frac{N_{j}}{N_{j b a s e}} \\
R_{x}=\sum_{x=1}^{X} \sigma_{x j} R_{j}
\end{gathered}
$$

In Equation 5, $R_{j}$ is the habitat scarcity index of landscape type $j . N_{j}$ is the number of grids of landscape type $j$ in the current or future map. $N_{j b a s e}$ is the number of grids of landscape type $j$ in the baseline landscape map. If there is no landscape type $j, R_{j}=$ 0 .

In Equation 6, $R_{x}$ is the habitat scarcity index. $\sigma_{x j}$ is the discriminant coefficient of the landscape type $j$. When the grid is the current landscape type $j, \sigma_{x j}=1$, otherwise $\sigma_{x j}=0$.

\section{Results}

We loaded the resulting image of the model run (including the habitat degradation raster map and the habitat quality raster map) into ArcGIS, analyze the degree of habitat degradation and habitat quality in each year by reclassifying images furtherly. It was possible to compare the degree of habitat degradation and the changes in habitat quality through grid calculation.

\section{Landscape changes}

From the perspective of landscape composition, the landscape types of the study area were mainly aquaculture pond, Phragmites australis marsh, Suaeda salsa marsh, Spartina alterniflora marsh and mudflat. Comparing the changes during 1983 to 2011 (Table 3), it could be found that the area of aquaculture pond, Phragmites australis marsh, and Spartina alterniflora marsh in the study area increased, while the area of Suaeda salsa marsh first increased and then decreased. Phragmites australis marsh increased from $19.62 \%$ in 1983 to $31.46 \%$ in 2011 , and the growth was faster and faster. Spartina alterniflora marsh increased from $1.69 \%$ in 1983 to $23.77 \%$ in 2011, and the expansion rate was getting faster and faster. The area percentage of Suaeda salsa marsh was $26.85 \%, 29.13 \%$ and $9.98 \%$ in 1983, 1997 and 2011, respectively, and it first increased and then decreased, and it decreased rapidly, the main reason for the increase was that Spartina alterniflora marsh had not formed a close interlaced zone with 
Suaeda salsa marsh, and there were original ecological evolution sequences of mudflat to Suaeda salsa marsh between 1983 and 1997. The sharp decrease between 1997 and 2011 was due to the two-way extrusion of Phragmites australis marsh and Spartina alterniflora marsh, and the area of Suaeda salsa marsh continued to shrink. Mudflat decreased from 46.93\% in 1983 to $17.63 \%$ in 2011, and the rate of reduction was also increasing faster, mainly due to the expansion of Suaeda salsa marsh and Spartina alterniflora marsh. The area of the aquaculture pond had changed greatly, from $0 \%$ in 1983 to $12.11 \%$ in 2011 . The road area had nearly doubled in 2011 compared with 1983. According to the two periods, it could be concluded that the effect of human activities on the study area was continuous and stable.

Table 3. Area changes of landscape types in the study area

\begin{tabular}{c|cc|cc|cc}
\hline \multirow{2}{*}{ Landscape types } & \multicolumn{2}{|c|}{1983} & \multicolumn{2}{c|}{1997} & \multicolumn{2}{c}{2011} \\
\cline { 2 - 7 } & $\begin{array}{c}\text { Area } \\
\left(\times 10^{2} \mathrm{hm}^{2}\right)\end{array}$ & $\begin{array}{c}\text { The percentage } \\
(\%)\end{array}$ & $\begin{array}{c}\text { Area } \\
\left(\times 10^{2} \mathrm{hm}^{2}\right)\end{array}$ & $\begin{array}{c}\text { The percentage } \\
(\%)\end{array}$ & $\begin{array}{c}\text { Area } \\
\left(\times 10^{2} \mathrm{hm}^{2}\right)\end{array}$ & $\begin{array}{c}\text { The percentage } \\
(\%)\end{array}$ \\
\hline Aquaculture pond & 0 & 0 & 11.67 & 6.06 & 23.32 & 12.10 \\
River & 6.41 & 3.33 & 4.16 & 2.16 & 4.11 & 2.13 \\
Mudflat & 90.39 & 46.93 & 63.72 & 33.08 & 33.95 & 17.62 \\
$\quad \begin{array}{c}\text { Road } \\
\begin{array}{c}\text { Phragmites } \\
\text { australis marsh }\end{array}\end{array}$ & 3.04 & 1.58 & 4.81 & 2.50 & 5.64 & 2.93 \\
$\begin{array}{c}\text { Spartina } \\
\text { alterniflora marsh }\end{array}$ & 37.79 & 19.62 & 42.65 & 22.14 & 60.6 & 31.46 \\
$\begin{array}{c}\text { Suaeda salsa } \\
\text { marsh }\end{array}$ & 51.25 & 1.69 & 10.51 & 5.46 & 45.8 & 23.77 \\
\hline
\end{tabular}

According to the area and number of landscape patches, it could be found that the number and area of landscape patches changed significantly from 1983 to 2011 and the number of patches increased from 53 to 291, an increase of $294.34 \%$; the average patch area decreased from $363.47 \mathrm{hm}^{2} 66.20 \mathrm{hm}^{2}$, a decrease of $81.79 \%$. The landscape fragmentation trend in the study area was obvious, and the landscape segmentation phenomenon was intensified. The contagion index and aggregation index were reduced from 61.50 and 96.05 to 45.84 and 89.36 , respectively. The mean shape index dropped from 1.59 to 1.30 , indicating that the landscape in the study area was gradually increased by human disturbance, and the landscape patch shape tended to be regular.

\section{Habitat degradation analysis}

The degree of habitat degradation had certain reference value in predicting the possibility of habitat damage in the future. The degree of habitat degradation directly reflected the threat of threat factors to landscape types and was directly proportional to them. The higher the degree of habitat degradation was, the greater the impact of the threat factors on the region would be. The core area of the Jiangsu Yancheng Wetland National Nature Reserve - Rare birds had a high degree of ecological environmental protection and a low degree of habitat degradation generally. However, in terms of time, habitat degradation was becoming more and more serious, and the value of habitat degradation index was increasing more and more quickly. The value of habitat degradation index was 0.006, 0.009 and 0.024, in 1983, 1997 and 2011, respectively. It can be seen from Table 4 that the value of habitat degradation index was most 
concentrated between 0 and 0.02 and the area percentage were $100 \%, 90.73 \%$ and $44.39 \%$ in 1983, 1997 and 2011, respectively. In 1983, the degree of habitat degradation was low, and the value of degradation index was all concentrated in the range of 0-0.02. In 1997, the value of habitat degradation was still low, but compared with the 1983, the area percentage in the range of $0.02-0.04$ was $9.27 \%$, the area with the value between 0.04-0.06 was very small. In 2011, the habitat degradation was intensified, and the area percentage of degradation ranging from 0 to 0.02 was less than half of that in 1997, the area ratio in the range of $0.02-0.04$ reached $30.19 \%$, the area ratio in the range of $0.04-0.06$ reached $22.99 \%$, the area ratio in the range of $0.06-0.08$ was $2.43 \%$, and the highest value of habitat degradation index reached 0.072 .

Table 4. Habitat degradation degree classification statistics from 1983 to 2011

\begin{tabular}{c|cc|cc|cc}
\hline \multirow{2}{*}{ Grading ranges } & \multicolumn{2}{|c|}{1983} & \multicolumn{2}{c|}{1997} & \multicolumn{2}{c}{2011} \\
\cline { 2 - 7 } & $\begin{array}{c}\text { Area } \\
\left(\times 10^{2} \mathrm{hm}^{2}\right)\end{array}$ & The percentage $(\%)$ & $\begin{array}{c}\text { Area } \\
\left(\times 10^{2} \mathrm{hm}^{2}\right)\end{array}$ & The percentage $(\%)$ & $\begin{array}{c}\text { Area } \\
\left(\times 10^{2} \mathrm{hm}^{2}\right)\end{array}$ & The percentage (\%) \\
\hline $0-0.02$ & 192.60 & 100 & 174.78 & 90.73 & 85.51 & 44.39 \\
$0.02-0.04$ & 0 & 0 & 17.85 & 9.27 & 58.17 & 30.19 \\
$0.04-0.06$ & 0 & 0 & 0.02 & 0 & 44.28 & 22.99 \\
$0.06-0.08$ & 0 & 0 & 0 & 0 & 4.69 & 2.43 \\
\hline
\end{tabular}

It can be seen from Fig. 2 that what had no habitat degradation or low degree of degradation were mainly threat factors themselves, namely Spartina alterniflora, roads, aquaculture ponds and rivers. The region with the higher value of habitat degradation index was mainly located in the neighborhood of the threat factors. From 1983 to 2011, as the intensity of threat factors increased, the area of the region with the high value of habitat degradation index also expanded, directly causing the value of habitat degradation index to become higher. In 1983, the areas with the high value of habitat degradation were mainly located on both sides of Xinyanggang River and Doulonggang River and the spatial differentiation of habitat degradation had obvious lateral belt distribution. In 1997, the area with the high value of habitat degradation was mainly distributed around the breeding ponds, the Doulonggang River, and the large Spartina alterniflora plaques in the south of the study area. In 2011, the areas with the high value of habitat degradation were mainly distributed in the interlaced belts between aquaculture pond and Phragmites australis marsh, Salsa marsh and Spartina marsh, mudflat and Spartina marsh. The spatial differentiation of habitat degradation had a distinct longitudinal band distribution.

\section{Habitat quality assessment}

During 1983 to 2011, the habitat quality of the core area of Yancheng Wetland National Nature Reserve was degraded. The value of habitat quality index decreased slightly from 0.863 to 0.846 during 1983 to 1997 , the habitat quality declined obviously from 1997 to 2011 , it fell from 0.846 to 0.742 , a decrease of $12.29 \%$.

The value of habitat quality index was divided into four sections: $0-0.25,0.25-0.5,0.5-$ 0.75 , and $0.75-1$, which indicated that the habitat quality grades were poor, medium, good, and excellent respectfully. It can be seen from Table 5 that the habitat quality of the study 
area was high generally, and the grade of habitat quality were mostly excellent, and then medium and poor successively, there was no distribution at the good grade. Thus, in terms of regional differences, the habitat quality in the study area showed a "polarization" trend. According to the changes of the area ratio in the three levels, it showed "two increasing and one decreasing". The area of the excellent habitat quality was in a state of decline, and the area ratio had decreased from $93.57 \%$ in 1983 to $59.08 \%$ in 2011 , the decline reached $36.86 \%$, and the decline from 1983 to 2011 was getting faster and faster. The areas of poor and medium habitat quality were increasing, and the area of poor habitat quality increased from $1.40 \%$ in 1983 to $2.91 \%$ in 2011 , which was more than doubled. The area of medium habitat quality increased from 5.03\% in 1983 to $38.01 \%$ in 2011 , an increase of more than 6.5 times, and the increasing speed of the areas of poor and medium habitat quality were becoming faster and faster.
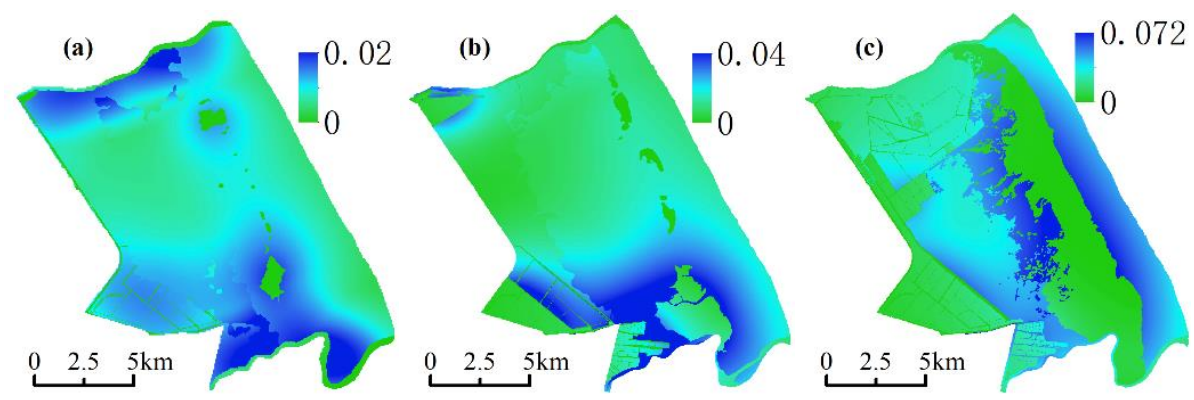

Figure 2. Habitat quality degradation index distribution maps. (a)It is a habitat quality degradation index distribution map in 1983. (b) It is a habitat quality degradation index distribution map in 1997. (c) It is a habitat quality degradation index distribution map in 2011

Table 5. Habitat quality classification statistics from 1983 to 2011

\begin{tabular}{c|cc|cc|cc}
\hline \multirow{2}{*}{ Grading ranges } & \multicolumn{2}{|c|}{1983} & \multicolumn{2}{c|}{1997} & \multicolumn{2}{c}{2011} \\
\cline { 2 - 6 } & $\begin{array}{c}\text { Area } \\
\left(\times 10^{2} \mathrm{hm}^{2}\right)\end{array}$ & The percentage (\%) & $\begin{array}{c}\text { Area } \\
\left(\times 10^{2} \mathrm{hm}^{2}\right)\end{array}$ & The percentage (\%) & $\begin{array}{c}\text { Area } \\
\left(\times 10^{2} \mathrm{hm}^{2}\right)\end{array}$ & The percentage (\%) \\
\hline $0-0.25$ & 2.70 & 1.40 & 2.77 & 1.44 & 5.61 & 2.91 \\
$0.25-0.50$ & 9.69 & 5.03 & 26.61 & 13.81 & 73.22 & 38.01 \\
$0.50-0.75$ & 0 & 0 & 0 & 0 & 0 & 0 \\
$0.75-1.00$ & 180.21 & 93.57 & 163.27 & 84.75 & 113.82 & 59.08 \\
\hline
\end{tabular}

From the spatial differentiation maps of habitat quality (Fig. 3) and the habitat quality change maps (Fig. 4), it can be seen that the areas with relatively poor habitat quality were mainly concentrated in roads, rivers, aquaculture ponds and Spartina alterniflora. Especially the expansion of the aquaculture pond and Spartina alterniflora led to a rapid increase of the area with lower-level habitat quality. The areas with relatively good habitat quality were mainly in the original ecological vegetation distribution area, including Phragmites australis marsh, Salsa marsh and mudflat. The area where the habitat quality changes were located in the landscape interlaced belt, that was, the area where the landscape type changes, including the Phragmites australis marsh--aquaculture pond 
interlaced belt, Salsa marsh-Spartina marsh interlaced belt, and mudflat-Spartina marsh interlaced belt.
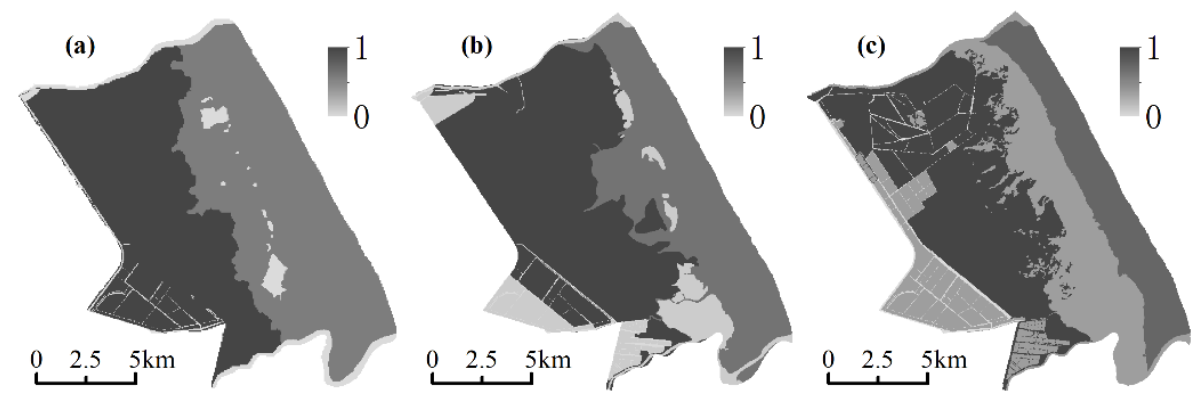

Figure 3. Habitat quality index distribution maps. (a) It is a habitat quality index distribution map in 1983. (b) It is a habitat quality index distribution map in 1997. (c) It is a habitat quality index distribution map in 2011
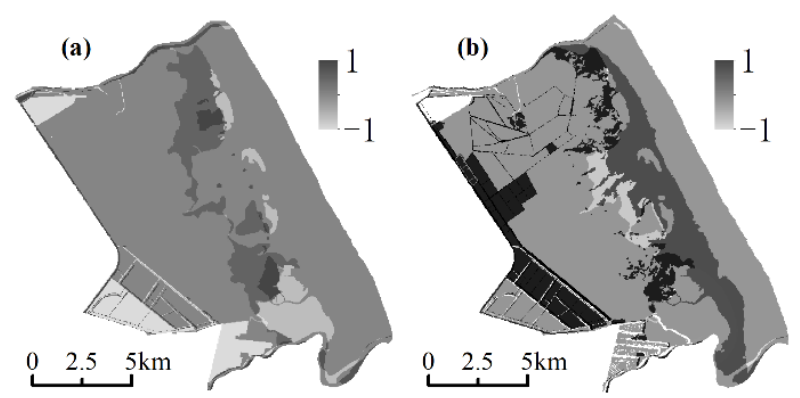

Figure 4. Spatial variation of habitat quality index. (a) It is the spatial variation of habitat quality index from 1983 to 1997. (b) It is the spatial variation of habitat quality index from 1997 to 2011

\section{Habitat scarcity evaluation}

The higher the scarcity index was, the more stable the overall ecosystem tended to be, and the ecosystem structure and function would decline. The lower the scarcity index was, the more fragile the ecosystem would be, but at the same time, it tended to be the more susceptible and need to strengthen protection. Based on the conditions of no introduction of Spartina alterniflora in the mudflat in 1983, the scarcity index was -0.10 in 1997, the scarcity index was 0.173 in 2011 , and the scarcity index was rising, indicating that the ecosystem was stable and the ecosystem function was declining when the region was in human activities and invasion of alien species. The value of $R_{j}$ was close to 1 and the current or future landscape type was more important to biodiversity conservation when the type of landscape was rare. The $R_{j}$ value of Salsa marsh and the mudflat were obviously rising, and they were closer to 1 . In 2011, the $R_{j}$ values of salsa marsh and the mudflat were the highest, 0.628 and 0.637 respectively, indicating that these landscape types were decreasing and became more and more scarce in the study area. They needed more protection and ecological restoration. 


\section{Discussion}

The spatial and temporal changes of habitat quality in the core area of Yancheng Wetland National Nature Reserve $\bullet$ Rare birds are mainly caused by human activities and the expansion of Spartina alterniflora. The study area focused on protecting the wintering habitat of Red-crowned cranes. In order to create good artificial habitats for rare species such as Red-crowned cranes, it began to establish some artificial wetlands in 1993, and implemented a large area of Phragmites australis marsh recovery work. Driven by economic interests, some of the projects had changed the original plan, and the area of Phragmites australis base and aquaculture pond were mainly based on economic development, had increased significantly. As a result, the area of aquaculture ponds in the study area has increased from $0 \%$ in 1983 to $12.10 \%$ in 2011 , and the increase in two periods almost the same, further illustrating the sustainability and stability of human activities. The aquaculture ponds were mainly developed from Phragmites australis marsh, and the original ecological landscape was transformed into a threat factor, which caused the habitat to degenerate and the habitat quality decreased. On the other hand, the artificial embankment not only generated a new threat factor, but also changed the hydrological process, blocked the intrusion of tidal water, effectively stored fresh water, further affected the soil hydrological properties, and accelerated the speed of the transformation of Suaeda salsa marsh to Phragmites australis marsh and changed the landscape structure of the wetlands.

In the 1980s, Spartina alterniflora was successfully introduced in the coastal of Jiangsu Province. After the formation of a large-area community in the 1990s, the area expanded rapidly, forming a coastal vegetation with a few kilometers wide and a northsouth coastal wetland. Although Spartina alterniflora had played an active role in protecting beaches, it was a threat factor for habitats of rare species such as Red-crowned cranes. On the one hand, the superior silt-promoting function of Spartina alterniflora resulted in a significant increase in the elevation of the beach surface and the thickness of the silt reached $1.5 \mathrm{~m}$ on the spot, which made it difficult for the tide to pass through Spartina alterniflora marsh and reach Suaeda salsa marsh. The habitat of Suaeda salsa caused significant degradation. On the other hand, on the lower edge of Suaeda salsa marsh, the settlement of Spartina alterniflora made the repeat of the niche, while the ecological amplitude characteristics of Spartina alterniflora and the sexual and asexual dual reproduction mode made it the inter-species competition was in a dominant position, and eventually Suaeda salsa marsh was constantly replaced by the Spartina alterniflora marsh. In the landscape composition of the study area, Suaeda salsa marsh, which is inhabited by rare species such as Red-crowned cranes, has been transformed into a habitat quality threat factor, resulting in a decline in habitat quality.

Human activities and the expansion of Spartina alterniflora have made the core area of Yancheng Wetland National Nature Reserve • Rare birds more threatening, ecosystem function decline, ecological restoration will become an important research topic in the region. At present, positive results have been achieved. The core area had basically completed "removing aquaculture ponds and restoring marshes" project, the buffer zone "removing aquaculture ponds and restoring marshes" was also actively carried out. However, the threat of Spartina alterniflora expansion is still growing and will continue to threaten the habitat quality of the study area. On the one hand, it is necessary to control the expansion of Spartina alterniflora, maintain a moderate scale, give full play to its revetment function, and reduce the threat to landscape habitats. On the other hand, it is 
necessary to actively restore Suaeda salsa marsh, restore the evolution sequence of mudflat to Suaeda salsa marsh, maintain biodiversity and improve regional habitat quality.

\section{Conclusion}

The landscape composition of the core area of Yancheng Wetland National Nature Reserve - Rare birds is mainly aquaculture pond, Phragmites australis marsh, Suaeda salsa marsh, Spartina alterniflora marsh and mudflat. From 1983 to 2011, the change of landscape composition showed that the aquaculture pond, Phragmites australis marsh and Spartina alterniflora marsh increased continuously, and the increasing speed became faster and faster. The area of mudflat decreased, and the area of Suaeda salsa marsh first increased and then decreased, and the corresponding decrease was faster and quicker. The landscape changes are mainly conversions of mudflat into Spartina alterniflora marsh and Suaeda salsa marsh, Suaeda salsa marsh into Phragmites australis marsh and Spartina alterniflora marsh and Phragmites australis marsh into culture pond.

From 1983 to 2011, although the habitat quality of the core area of Yancheng Wetland National Nature Reserve - Rare birds were at the excellent level, the degradation trend was also obvious. From 1997 to 2011, the value of habitat quality index decreased from 0.846 to 0.742 , a decrease of $12.29 \%$. The areas with relatively poor habitat quality mainly concentrate on the distribution areas of threat factors such as Spartina alterniflora marsh, roads, aquaculture ponds, rivers, etc. The area with relatively good habitat quality mainly distributed in the original ecological vegetation distribution areas, including Phragmites australis marsh, Suaeda salsa marsh and mudflat; and the area of habitat quality change were located in the landscape ecotone. The value of habitat degradation index was increasing. The spatial heterogeneity of the habitat degradation was characterized by a transition from a transverse strip distribution to a longitudinal strip distribution. The habitat scarcity index increased significantly, indicating that the ecosystem of the study area was stable and the ecosystem function was declining.

The degradation of coastal wetland habitat seriously threatened the habitat of Red-crowned Crane and other rare species. Yancheng coastal wetland had joined the World Heritage List, and scientific protection and restoration is imminent. According to the characteristics of Yancheng coastal wetland, systematically analyzing the degradation mechanism of coastal wetland habitat quality, clarifying the mechanism of ecological restoration from the perspective of landscape structure and ecological process, reconstructing and optimizing regional habitat ecological protection network, and carrying out ecological restoration technology integration and demonstration in typical areas need to be strengthened in the future research.

Acknowledgements. This research was supported by National Natural Science Foundation of China (No.41771199 and No.41501567) and the Natural Science Foundation of Jiangsu Province, China (Grants No. BK20171277), Natural Science Research Projects of Colleges and Universities in Jiang Province (No.18KJD170001) and Qing Lan Project in Jiangsu University of China. We acknowledged Yancheng Natural Reserve Management Office for giving us the opportunity to enter the study area and carry out the field works gratefully. 


\section{REFERENCES}

[1] Bai, J., Liu, J., Yu, K. Y., Zhang, L. B., Li, X. T., Wang, D. W. (2015): Assessment of tenyear change of habitat quality in Minjiang river basin based on InVEST-Biodiversity. Forestry research and sketch (3): 5-12.

[2] Bao, Y. B., Liu, K., Li, T., Hu, S. (2015): Effects of land use change on habitat based on InVEST Model-Taking Yellow River Wetland Nature Reserve in Shanxi Province as an example. - Arid Zone Research 32(3): 622-629.

[3] Bhagabati, N. K., Ricketts, T., Sulistyawan, T. B. S., Conte, M., Ennaanay, D., Hadian, O. (2014): Ecosystem services reinforce sumatran tiger conservation in land use plans. Biological Conservation 169(387): 147-156.

[4] Chaplinkramer, R., Sharp, R. P., Mandle, L., Sim, S., Johnson, J., Butnar, I. (2015): Spatial patterns of agricultural expansion determine impacts on biodiversity and carbon storage. Proceedings of the National Academy of Sciences of the United States of America 112(24): 7402-7407.

[5] Chen, Y., Qiao, F., Jiang, L. (2016): Effects of Land Use Pattern Change on Regional Scale Habitat Quality Based on InVEST Model-a Case Study in Beijing. - Acta Scientiarum Naturalium Universitatis Pekinensis 52(3): 553-562.

[6] Chu, L., Huang, C., Liu, Q. S., Liu, G. H. (2016): Changes of coastal zone landscape spatial patterns and ecological quality in Liaoning Province from 2000 to 2010. - Resources Science 37(10): 1962-1972.

[7] Goldstein, J. H., Caldarone, G., Duarte, T. K., Ennaanay, D., Hannahs, N., Mendoza, G. (2012): Integrating ecosystem-service tradeoffs into land-use decisions. - Proceedings of the National Academy of Sciences of the United States of America 109(19): 7565-7570.

[8] Gong, J., Ma, X. C., Zhang, L. L., Liu, D. Q., Zhang, J. X. (2018): Spatiotemporal variation of habitat quality in Bailongjiang watershred in Gansu based on InVEST MODEL. Research of Soil and Water Conservation 25(3): 191-196.

[9] Guo, J., Xu, M., Chen, J. H., Xie, T. Z., Li, Y. Q., Gong, G. T., Qing, G., Mu, C. L. (2016): Evaluation on habitat quality in arid and semi-arid valley area of the upper reaches of the Minjiang River-Using the Zagunao River as an Example. - Journal of Sichuan Forestry Science and Technology 37(3): 25-30.

[10] Hall, L. S., Krausman, P. R., Morrison, M. L. (1997): The habitat concept and a plea for standard terminology. - Wildlife Society Bulletin 25(1): 173-182.

[11] Li, Y., Zhang, F., Yang, H. J., Yin, S. J., Hong, Y. F., Zhu, H. T., Teng, J. H., Dai, Y. T. (2016): On habitat quality change monitoring by remote sensing in eco-functional area of biological diversity. - Environment and Sustainable Development 41(2): 46-48.

[12] Liu, D. W., Zhang, Y. L., Sun, Y., Lu, S. C., Cheng, H., Mu, S. J., Lu, C. H. (2016): Population dynamics and habitat selection of overwintering Red-Crowned Cranes in coastal wetland of Yancheng, Jiangsu Province. - Journal of Ecology and Rural Environment 32(3): 473-477.

[13] Mdk, L., Matlock, M. D., Cummings, E. C., Nalley, L. L. (2013): Quantifying and mapping multiple ecosystem services change in west Africa. - Agriculture Ecosystems \& Environment 165(1751): 6-18.

[14] Meng, X., Ren, Z. Y., Zhang, C. (2012): Study on land use change and ecological risk in Xianyang city. - Arid Zone Research 29(1): 137-142.

[15] Nelson, E., Mendoza, G., Regetz, J. (2009): Modeling multiple ecosystem services, biodivrsity conservation, commodity production, and tradeoffs at landscape scales. - Front Ecology and Environment 7(1): 4-11.

[16] Ou, W. X., Yuan, W. J. (2015): Priority of red-crowned crane wintering habitat patches using landscape connectivity in the Yancheng coastal wetland. - Resources Science 37(4): 823-831. 
[17] Sharp, R., Kramer, R. C., Wood, S., Guerry, A., Tallis, H. T., Ricketts, T. (2017): InVEST version user's guide. - The Natural Capital Project, Stanford University, University of Minesota, The Nature Consercation, World Wildlife Fund.

[18] Sun, Y. T., Zhang, J. C. (2011): The ecological evaluation of north branch of Changjiang estuary wetland nature reserve. - Wetland Science \& Management 7(1): 25-28.

[19] Sun, X. B., Liu, H. Y. (2011): Temporal changes of Grus japonensis suitable wintering habitat in coastal zone of Yancheng, Jiangsu. - Chinese Journal of Ecology 30(4): 694-699.

[20] Terrado, M., Sabater, S., Chaplinkramer, B., Mandle, L., Ziv, G. (2016): Model development for the assessment of terrestrial and aquatic habitat quality in conservation planning. - Science of the Total Environment 540(1): 63-70.

[21] Wang, H. J. (2016): Evaluation of the Ecological Quality for Sanjiangyuan based on InVEST. - Value Engineering 35(12): 66-70.

[22] Wang, H., Liang, H. M., Xu, Y. L., Yang, Y. F., Ouyang, Q. (2015): Ecological suitability analysis and functional zoning of rare bird wetland in Yancheng. - Journal of forestry engineering 29(4): 145-149.

[23] Wu, J. S., Cao, Q. W., Shi, S. Q., Huang, X. L., Lu, Z. Q. (2015): Spatio-temporal variability of habitat quality in Beijing-Tianjin-Hebei Area based on land use change. - Chinese Journal of Applied Ecology 26(11): 3457-3466.

[24] Xiao, M. (2011): GIS Application in evaluation of ecological environment quality -- taking the lower reaches of Changhua river as an example. - Doctoral dissertation, Hainan University.

[25] Yang, J., Shan, L. Z., Xi, J. C., Li, X. M., Ge, Q. S. (2014): Land use pattern changes and ecological effects in Nansihu wetland. - Resources Science 36(4): 856-864.

[26] Yuan, L., Gong, W. F., Yu, C. L. (2009): Spatial-temporal changes of habitats for redcrowned cranes in Zhalong wetland based on RS and Gis. - Journal of Northeast Forestry University 37(8): 34-38.

[27] Zhang, Y., Xie, Y. C., Qi, S. S., Gong, J., Zhang, L. L. (2016): Carbon storage and spatial distribution characteristics in the Bailongjiang Watershed in Gansu based on InVEST model. - Resources Science 38(8): 1585-1593.

[28] Zhang, J., Liao, T. J., Huang, J. L., Zhou, L. (2017): Research of habitat quality in Wanzhou district. - Human Agriculture Sciences (1): 45-50.

[29] Zhang, D. Z., Sun, X. Y., Yuan, X. Z., Liu, F., Guo, H. W., Xu, Y., Li, B. F. (2018): Land use change and its impact on habitat quality in Lake Nansi Basin from 1980 to 2015. Journal of Lake Sciences 30(2): 349-357.

[30] Zhao, M. Y., Xue, L. (2017): Monitoring by remote sensing of habitat quality changes in Xianyang city. - Remote Sensing Technology and Application 32(6): 1171-1180.

[31] Zhong, L., Wang, J. (2017): Evaluation on effect of land consolidation on habitat quality based on InVEST model. - Transactions of the Chinese Society of Agricultural Engineering 33(1): 250-255. 